\title{
A FREE BOUNDARY FOCUSING PROBLEM
}

\author{
DANIELLE HILHORST AND JOSEPHUS HULSHOF
}

(Communicated by Barbara Lee Keyfitz)

\begin{abstract}
We consider a one-dimensional free boundary problem arising in combustion theory and establish that all solutions are asymptotically equal to a similarity solution which vanishes in a finite time.
\end{abstract}

\section{INTRODUCTION}

In this paper we deal with the problem

$$
\begin{cases}u_{t}=u_{x x} & \text { for } 0<x<\zeta(t), t>0 ; \\ u_{x}(0, t)=0 & \text { for } t>0 ; \\ u(\zeta(t), t)=0 & \text { for } t>0 ; \\ u_{x}(\zeta(t), t)=1 & \text { for } t>0 ; \\ u(x, 0)=u_{0}(x) \leq 0 & \text { for } 0 \leq x \leq \zeta_{0} \\ \zeta(0)=\zeta_{0}, & \end{cases}
$$

where the unknowns are the functions $u(x, t)$ and $\zeta(t)$ and where $\zeta_{0}>0$ and the nonpositive function $u_{0} \in C\left(\left[0, \zeta_{0}\right]\right)$ are the given initial data.

Problem $(\mathrm{P})$ is a free boundary problem for the one-dimensional linear heat equation, the free boundary being the curve $x=\zeta(t)$, which is also called the interface. At this interface one has two boundary conditions, which makes it, in general, impossible to have a fixed boundary.

This problem is a one-dimensional version of a more-dimensional free boundary problem arising in the theory of flame propagation, in which both $u$ and its normal derivative are prescribed at the interface (see, e.g., [BL]). For this general and considerably more difficult case there is a paper by Berestycki, Caffarelli, and Nirenberg on a slightly different problem about travelling wave solutions and a very recent preprint by Caffarelli and Vazquez [CV] in which existence and regularity of solutions to the initial value problem are established. The

Received by the editors November 30, 1992.

1991 Mathematics Subject Classification. Primary 35K55, 35K55, 80A25.

Key words and phrases. Free boundary problem, similarity variables, maximum principle, asymptotic behaviour.

This research was supported by EEC-contract SC1-0019-C-(TT) and by N.W.O., the Nederlandse Stichting voor Wetenschappelijk Onderzoek (project: "Nonlinear Transport Phenomena in Porous Media"). 
uniqueness question, however, is still open. Besides the question of local existence and uniqueness of a solution, say on a maximal time interval $[0, T)$, one is interested in the behaviour as $t \rightarrow T$. We note that in this problem $T$ is usually a finite number.

Assuming that $u_{0}$ is not identically equal to zero in a neighbourhood of $\zeta_{0}$, existence and uniqueness results of (weak) solutions for problem (P) follow from the theory of elliptic-parabolic equations $[\mathrm{H}]$. (We remark here that the radially symmetric case can also be handled by this theory but that involves a lot more work.) Moreover, it follows from the same theory [HH] that both $u$ and $\zeta$ are smooth for $0<t<T$ and, also, by the maximum principle, that $u$ is negative. The maximal value of $T$ follows explicitly from the initial data, in view of the following conservation law. Integrating the equation with respect to $x$ we have

$$
\frac{d}{d t} \int_{0}^{\zeta(t)} u(x, t) d x=1
$$

so

$$
T=-\int_{0}^{\zeta_{0}} u_{0}(x) d x
$$

It was shown in $[\mathrm{H}]$ that

$$
\lim _{t \rightarrow T} \zeta(t)=0,
$$

i.e., the solution focuses at time $t=T$. In the context of the elliptic-parabolic problem, which models saturated-unsaturated flows in porous media, $T$ is called the saturation time. We shall prove that as $t \rightarrow T$, the solution is asymptotically self-similar. To do so we observe that $(\mathrm{P})$ has self-similar solutions of the form

$$
u(x, t)=\sqrt{T-t} f(\eta), \quad \eta=\frac{x}{\sqrt{T-t}},
$$

where $f(\eta)$ has to satisfy

$$
\begin{cases}f^{\prime \prime}+\frac{1}{2} f=\frac{1}{2} \eta f^{\prime} \quad \text { for } 0 \leq \eta \leq a \\ f^{\prime}(0)=f(a)=0, & f^{\prime}(a)=1,\end{cases}
$$

for some $a>0$. Clearly (S) has exactly one solution pair $(f, a)$ with $f<0$ on $[0, a)$. This yields a similarity solution of $(\mathrm{P})$ which "focuses" at time $t=T$.

In order to state our results we transform $(P)$ by setting

$$
\begin{array}{ll}
\tau=-\log (T-t), & \eta=\frac{x}{\sqrt{T-t}}, \\
w(\eta, \tau)=\frac{u(x, t)}{\sqrt{T-t}}, & \psi(\tau)=\frac{\zeta(t)}{\sqrt{T-t}} .
\end{array}
$$

Then, if $(u, \zeta)$ is a solution of $(\mathrm{P})$ on $(0, T)$ and $T$ is given by (1.2), the pair 
$(w, \psi)$ is a solution of

$$
\begin{cases}w_{\tau}=w_{\eta \eta}-\frac{1}{2} \eta w_{\eta}+\frac{1}{2} w & \text { for } 0<\eta<\psi(\tau),-\log T<\tau<\infty \\ w_{\eta}(0, \tau)=0 & \text { for }-\log T<\tau<\infty \\ w(\psi(\tau), \tau)=0 & \text { for }-\log T<\tau<\infty \\ w_{\eta}(\psi(\tau), \tau)=1 & \text { for }-\log T<\tau<\infty \\ w(\eta,-\log T)=w_{0}(\eta) \leq 0 & \text { for } 0 \leq \eta \leq \psi_{0} \\ \psi(-\log T)=\psi_{0}, & \end{cases}
$$

where $w_{0}$ and $\psi_{0}$ are the transformed initial data.

Theorem 1.1. Let $u_{0} \in C\left(\left[0, \zeta_{0}\right]\right)$ be nonpositive, not identically equal to zero in a neighbourhood of $\zeta_{0}>0$; let $(u, \zeta)$ be a solution of $(\mathrm{P})$; let $w$ and $\psi$ be defined by (1.5), where $T$ is given by (1.2); and let $(f, a)$ be the unique solution of (S) with $f$ negative. Then $\lim _{\tau \rightarrow \infty} \psi(\tau)=a$, and $w(\eta, \tau) \rightarrow$ $f(\eta)$ uniformly as $\tau \rightarrow \infty$.

\section{UNIFORM BOUNDS ON $\psi(\tau)$}

Throughout this section we assume that $u_{x}$ and $w_{\eta}$ satisfy

$$
0 \leq u_{x}=w_{\eta} \leq L
$$

for some fixed constant $L>0$. We notice that the upper bound follows from applying the maximum principle to $u_{x}$, starting at any positive $t$-value. The zero lower bound is less obvious and only holds for $t$ sufficiently close to $T$. This was proved in $[\mathrm{H}]$.

Our first step is to show that the transformed solution has a free boundary which stays away from zero and infinity. Note that the transformed solution has

$$
\int_{0}^{\psi(\tau)} w(\eta, \tau) d x=-1=\int_{0}^{a} f(\eta) d \eta
$$

Solutions $w$ with a larger integral (less negative) correspond to solutions $u$ which focus earlier, so $w$ focuses in finite time, whereas solutions $w$ with a smaller integral (more negative) correspond to solutions $u$ which focus later, so $w$ exists globally with an interface escaping to infinity. Unless otherwise stated, the solutions we consider satisfy (2.2).

We begin with a lower bound for the third-order space derivative.

Lemma 2.1. There exists $M>0$ such that $w_{\eta \eta \eta} \geq-M e^{-\tau}$, for all $\tau$ sufficiently large.

Proof. Let $z=u_{x x x}$. Then $z$ satisfies $z_{t}=z_{x x}$, and $z(0, t)=u_{x t}(0, t)=0$. On the interface we have, differentiating the free boundary conditions with respect to $t$,

$$
u_{x}(\zeta(t), t) \zeta^{\prime}(t)+u_{t}(\zeta(t), t)=0, \quad u_{x x}(\zeta(t), t) \zeta^{\prime}(t)+u_{x t}(\zeta(t), t)=0
$$

so

$$
\begin{aligned}
z(\zeta(t), t) & =u_{x t}(\zeta(t), t)=-u_{x x}(\zeta(t), t) \zeta^{\prime}(t) \\
& =-u_{t}(\zeta(t), t) \zeta^{\prime}(t)=u_{x}(\zeta(t), t) \zeta^{\prime}(t)^{2}=\zeta^{\prime}(t)^{2} \geq 0
\end{aligned}
$$


Thus, by the maximum principle, and because the solution is smooth, $u_{x x x}$ is bounded from below if $t<T$ is away from zero. Since

$$
w_{\eta \eta \eta}=(T-t) u_{x x x}=e^{-\tau} u_{x x x}
$$

the lemma follows.

Lemma 2.2. $\liminf \operatorname{in}_{\tau \rightarrow \infty} \psi(\tau)<\infty$.

Proof. Suppose not; then $\psi(\tau) \rightarrow \infty$ as $\tau \rightarrow \infty$, so in particular $\psi(\tau)>a$ for all large values of $\tau \geq \tau_{0}$, for some $\tau_{0}$. However, away from the interface $w$ is strictly negative, so $w(a, \tau)<0$, and, moreover, there exists $\varepsilon>0$ such that

$$
\varepsilon f(\eta)>w\left(\eta, \tau_{0}\right) \text { for all } \eta \in[0, a j .
$$

The maximum principle implies that

$$
\varepsilon f(\eta) \geq w(\eta, \tau) \text { for all } 0 \leq \eta \leq a, \tau_{0} \leq \tau<\infty,
$$

and consequently $w(0, \tau)$ is bounded away from zero. Also, in view of (2.1) and (2.2), $w$ is uniformly bounded from below. Hence there exists a sequence $\tau_{n} \rightarrow \infty$ such that

$$
w_{\tau}\left(0, \tau_{n}\right) \rightarrow 0 \text { and } w\left(0, \tau_{n}\right) \rightarrow-b<0,
$$

whence, using the equation for $w$,

$$
w_{\eta \eta}\left(0, \tau_{n}\right) \rightarrow \frac{b}{2}
$$

Because of Lemma 2.1, we then have

$$
w\left(\eta, \tau_{n}\right) \geq-2 b+\frac{b}{8} \eta^{2}-\frac{1}{6} M e^{-\tau_{n}} \eta^{3},
$$

for $n$ large. This makes it impossible for $\psi\left(\tau_{n}\right)$ to become unbounded, which is a contradiction.

Lemma 2.3. There exists a $\tau_{0}$ such that $w_{\eta \eta}>0$ for all $\tau \geq \tau_{0}$ and for all $0 \leq \eta \leq \psi(\tau)$.

Proof. Since $w_{\eta \eta}$ and $u_{t}=u_{x x}$ have the same sign, it is sufficient to show that $u_{t}>0$ for $t$ close to $T$. For $q=u_{t}$ we have $q_{t}=q_{x x}$ while $q_{x}(0, t)=$ $u_{x t}(0, t)=0$ and, by (2.4), $q_{x}(\zeta(t), t)=u_{x t}(\zeta(t), t)=\zeta^{\prime}(t)^{2} \geq 0$. Thus the lemma will follow from the maximum principle if we can find a $t_{0} \in(0, T)$ such that $u_{x x}\left(x, t_{0}\right)>0$ for all $x \in\left[0, \zeta\left(t_{0}\right)\right)$, which is equivalent to $w_{\eta \eta}\left(\eta, \tau_{0}\right)>0$ for all $\eta \in\left[0, \psi\left(\tau_{0}\right)\right)$ for some sufficiently large $\tau_{0}$. We shall show that such a $\tau_{0}$ indeed exists.

Since

$$
1=-\int_{0}^{\psi(\tau)} w(\eta, \tau) d \eta \leq-w(0, \tau) \psi(\tau),
$$

we have by Lemma 2.2 that

$$
\liminf _{\tau \rightarrow \infty} w(0, \tau)<0 .
$$

We claim that this implies the existence of a number $\gamma>0$ and a sequence $\tau_{n} \rightarrow \infty$ such that

$$
w\left(0, \tau_{n}\right) \rightarrow-\gamma
$$


and

$$
\lim _{n \rightarrow \infty} w_{\tau}\left(0, \tau_{n}\right) \geq 0
$$

This can easily be seen by distinguishing between

$$
\liminf _{\tau \rightarrow \infty} w(0, \tau)=\limsup _{\tau \rightarrow \infty} w(0, \tau)<0
$$

and

$$
\liminf _{\tau \rightarrow \infty} w(0, \tau)<\limsup _{\tau \rightarrow \infty} w(0, \tau) \leq 0 .
$$

Combining (2.12) and (2.13) with the equation for $w$ we also have that

$$
\liminf _{n \rightarrow \infty} w_{\eta \eta}\left(0, \tau_{n}\right)>0 \text {. }
$$

Together with Lemma 2.1 again this implies, as in the proof of Lemma 2.2, that $\psi\left(\tau_{n}\right)$ is bounded and that

$$
w_{\eta \eta}\left(\eta, \tau_{n}\right)>0 \text { on }\left[0, \psi\left(\tau_{n}\right)\right],
$$

for large $n$. This completes the proof.

Corollary 2.4. $-w(0, \tau) \psi(\tau)>1>-\frac{1}{2} w(0, \tau) \psi(\tau)$ for all $\tau \geq \tau_{0}$.

Lemma 2.5. The functions $\psi(\tau)$ and $-w(0, \tau)$ remain bounded away from zero and infinity.

Proof. In view of Corollary 2.4 and the boundedness of $w$, the only thing we have to exclude is that there exists a sequence $\tau_{n} \rightarrow \infty$ such that

$$
w_{\tau}\left(0, \tau_{n}\right) \geq 0 \text { and } w\left(0, \tau_{n}\right) \rightarrow 0 \text { as } n \rightarrow \infty \text {. }
$$

Then

$$
w_{\eta \eta}\left(0, \tau_{n}\right)=w_{\tau}\left(0, \tau_{n}\right)-\frac{1}{2} w\left(0, \tau_{n}\right) \geq-\frac{1}{2} w\left(0, \tau_{n}\right)>0 .
$$

Hence, by Lemma 2.1 again,

$$
w\left(\eta, \tau_{n}\right) \geq w\left(0, \tau_{n}\right)-\frac{1}{4} w\left(0, \tau_{n}\right) \eta^{2}-\frac{M}{6} e^{-\tau_{n}} \eta^{3} .
$$

Since

$$
\begin{aligned}
0 & =\frac{d}{d \tau} w(\psi(\tau), \tau)=w_{\eta}(\psi(\tau), \tau) \psi^{\prime}(\tau)+w_{\tau}(\psi(\tau), \tau) \\
& =\psi^{\prime}(\tau)+w_{\eta \eta}(\psi(\tau), \tau)-\frac{1}{2} \psi(\tau),
\end{aligned}
$$

it follows from Lemma 2.3 that for some $K>0$,

$$
\psi(\tau) \leq K e^{\frac{1}{2} \tau}
$$

whence, by Corollary 2.4 ,

$$
-w(0, \tau) \geq \frac{1}{K} e^{-\frac{1}{2} \tau}
$$

Rewriting (2.20) as

$$
\frac{w\left(\eta, \tau_{n}\right)}{-w\left(0, \tau_{n}\right)} \geq-1+\frac{1}{4} \eta^{2}+\frac{M e^{-\tau_{n}}}{6 w\left(0, \tau_{n}\right)} \eta^{3}
$$


we see that because of (2.23) the coefficient of $\eta^{3}$ in (2.24) goes to zero at least as fast as $e^{-\tau / 2}$. This implies that $\psi\left(\tau_{n}\right)$ must be a bounded sequence, which contradicts (2.18) in view of Corollary 2.4.

\section{MONOTONICITY PROPERTIES OF $\psi(\tau)$}

In view of Lemma 2.3 it is no restriction to assume that, in addition to (2.1), (2.2), and Lemma 2.5,

$$
w_{\eta \eta}>0 \text {. }
$$

Theorem 3.1. The function $\psi(\tau)$ is eventually a monotone function of $\tau$, and its limit for $\tau \rightarrow \infty$ exists in $(0, \infty)$.

The proof is based on an application of lap number type arguments to the functions $w_{\tau}$ and $p=w_{\eta \tau}$ (see, e.g., $[\mathrm{M}]$ ).

Definition 3.2. Let $I \subset \Re$ be an interval, and let $f \in C(I)$. The number $l_{I}(f)$ is defined as the supremum, possibly infinity, of all integers $n$ for which there exist $x_{0}<x_{1}<x_{2}<\cdots<x_{n}, \quad x_{0}, \ldots, x_{n} \in I$, such that $f\left(x_{i-1}\right) f\left(x_{i}\right)<$ $0 \forall i=1, \ldots, n$.

Observe that $p$ satisfies the uniformly parabolic equation

$$
p_{\tau}=p_{\eta \eta}-\frac{1}{2} \eta p_{\eta}
$$

which does not contain terms of order zero. At the fixed boundary we have

$$
p(0, \tau)=0,
$$

whereas at the free boundary (see $(2.21)$ ),

$$
w_{\tau}(\psi(\tau), \tau)=-\psi^{\prime}(\tau),
$$

and also, differentiating the free boundary condition for $w_{\eta}$,

$$
p(\psi(\tau), \tau)=w_{\eta \tau}(\psi(\tau), \tau)=-\psi^{\prime}(\tau) w_{\eta \eta} .
$$

Because of (3.1) this implies in particular that at the free boundary $p=w_{\eta \tau}$ and $w_{\tau}$ have the same sign and can only disappear simultaneously.

Lemma 3.3. Let $l_{\tau}=l_{[0, \psi(\tau))}\left(w_{\tau}(\cdot, \tau)\right)$. Suppose that for some $\tau_{1}>\tau_{0}$ there exists $\tau_{2} \in\left(\tau_{0}, \tau_{1}\right)$ such that $\psi^{\prime} \neq 0$ on $\left(\tau_{2}, \tau_{1}\right)$. Then $l_{\tau_{1}}<\infty$.

Proof. We first observe that solutions of the linear heat equation are analytic in the space variable. Consequently, the functions $w_{\tau}$ and $p$ are analytic in $\eta$, so on any subinterval $[0, a] \subset[0, \psi(\tau))$ their numbers of sign changes are finite. In view of (3.5) it follows that $l_{t}<\infty$ for every $\tau \in\left(\tau_{2}, \tau_{1}\right)$.

Now suppose that $l_{\tau_{1}}=\infty$. Then also the number of sign changes of $p\left(\cdot, \tau_{1}\right)=w_{\eta \tau}\left(\cdot, \tau_{1}\right)$ is infinite, so there exist $0<\eta_{0}<\eta_{1}<\eta_{2}<\eta_{3}<\cdots \uparrow$ $\psi\left(\tau_{1}\right)$, such that $p\left(\eta_{i-1}, \tau_{1}\right) p\left(\eta_{i}, \tau_{1}\right)<0$ for all $i=1,2,3, \ldots$, and moreover, by Sard's Theorem [GP], we can choose the sequence $\eta_{i}$ in such a way that $p\left(\eta_{i}, \tau_{1}\right)$ is a regular value of both $p\left(\cdot, \tau_{1}\right)$ and $p(\cdot, \cdot)$ for all $i=$ $0,1,2,3, \ldots$. This implies that the level sets of $p$ going through the points $\left(\eta_{i}, \tau_{1}\right)$ are smooth curves which are locally (near $\left.\tau_{1}\right)$ of the form $\eta=\psi_{i}(\tau)$, with $\psi(\tau)$ smooth. By a straightforward application of the strong maximum 
principle it follows that these level curves, as long as they do not hit the lateral boundary, can be continued backward in $\tau$-time and remain of the same form. Clearly they cannot hit $\eta=0$, where $p=0$. At the free boundary we have that $p(\psi(\tau), \tau) \neq 0$ for every $\tau \in\left(\tau_{2}, \tau_{1}\right)$. Consequently every other curve, say those with $i$ odd, cannot hit the free boundary while $\tau \in\left(\tau_{2}, \tau_{1}\right)$. But then the same holds for the curves with $i$ even. Thus all $\psi_{i}(\tau)$ are defined for all $\tau \in\left(\tau_{2}, \tau_{1}\right)$. This contradicts the observation at the beginning of this proof.

Lemma 3.4. Suppose that for some $\eta_{1} \in\left(0, \psi\left(\tau_{1}\right)\right)$ we have $w_{\tau}\left(\cdot, \tau_{1}\right) \leq 0$ on $\left(\eta_{1}, \psi\left(\tau_{1}\right)\right)$ and $w_{\tau}\left(\eta_{1}, \tau_{1}\right)<0$. Then there exists $\tau_{3} \in\left(\tau_{1}, \infty\right)$ such that $w_{\tau}<0$ on the set $\left\{(\eta, \tau): \eta_{1} \leq \eta \leq \psi(\tau), \tau_{1}<\tau \leq \tau_{3}\right\}$. A similar statement holds for the case of reversed inequalities.

Proof. We shall first give the proof under the assumption that

$$
w_{\tau}\left(\cdot, \tau_{1}\right)<0 \text { on }\left[\eta_{1}, \psi\left(\tau_{1}\right)\right] \text {. }
$$

Choose $\tau_{3}>\tau_{1}$ in such a way that $w_{\tau}\left(\eta_{1}, \cdot\right)<0$ on $\left[\tau_{1}, \tau_{3}\right]$. We claim that the statement of the lemma holds for this choice of $\tau_{3}$.

Suppose not; then there exists a minimal $\tau_{4} \in\left[\tau_{1}, \tau_{3}\right)$ for which the statement is false. By continuity $\tau_{4}>\tau_{1}$, and by the maximum principle applied to $w_{\tau}$, it follows that $w_{\tau}<0$ on $\left\{(\eta, \tau): \eta_{1} \leq \eta<\psi(\tau), \tau_{1}<\tau \leq \tau_{4}\right\}$, and that $w_{\tau}\left(\psi\left(\tau_{4}\right), \tau_{4}\right)=0$, for otherwise $\tau_{4}$ is not minimal. Hence $\psi^{\prime}\left(\tau_{4}\right)=0$, and the Boundary Point Lemma applied to $w_{\tau}$ at the point $\left(\psi\left(\tau_{4}\right), \tau_{4}\right)$ implies that $w_{\eta \tau}\left(\psi\left(\tau_{4}\right), \tau_{4}\right)>0$, which is impossible in view of $(3.5)$.

In the case that the stronger assumption (3.6) does not hold, we can approximate $w\left(\cdot, \tau_{1}\right)$ uniformly in $C^{2}\left(\left[0, \psi\left(\tau_{1}\right)\right]\right)$ by a sequence of smooth functions $w_{0 n}$ such that $w_{0 n}^{\prime}\left(\psi\left(\tau_{1}\right)\right)=1, w_{0 n}^{\prime}(0)=w_{0 n}\left(\psi\left(\tau_{1}\right)\right)=0$, and

$$
w_{0 n}^{\prime \prime}(\eta)-\frac{1}{2} \eta w_{0 n}^{\prime}(\eta)+\frac{1}{2} w_{0 n}(\eta)<0 \text { for } \eta_{1} \leq \eta \leq \psi\left(\tau_{1}\right) \text {. }
$$

For every fixed $n$ we then have a solution $w_{n}$ of $(\tilde{\mathbf{P}})$ with initial data at $\tau_{1}$ given by $w_{0 n}$. This solution may be obtained by first transforming $(\tilde{\mathbf{P}})$ back into (P) again. From $[\mathrm{BH}]$ we know that the corresponding solutions $u_{n}$ have firstorder derivatives continuous up to the parabolic (free) boundary, and which are bounded uniformly in $n$. Consequently the solutions $u$ and $w$ are the uniform limits of the sequences $u_{n}$ and $w_{n}$, respectively, and interior and boundary estimates imply that, on the vertical segment $\left\{\eta_{1}\right\} \times\left[\tau_{1}, \tau_{3}\right], w_{n \tau}$ also converges uniformly to $w_{\tau}$. Hence by continuity $w_{n \tau}<0$ on this segment for all large $n$.

Because of (3.7) all $w_{n}$ satisfy (3.6). Hence by the first part of this proof, the statement of the lemma holds for all $w_{n}$. Consequently we have for $w$ that $w_{\tau} \leq 0$ on $\left\{(\eta, \tau): \eta_{1} \leq \eta \leq \psi(\tau), \tau_{1}<\tau \leq \tau_{3}\right\}$. However, the maximum principle implies again that $w_{\tau}<0$ on $\left\{(\eta, \tau): \eta_{1} \leq \eta<\psi(\tau), \tau_{1} \leq \tau \leq \tau_{3}\right\}$, and exactly as in the first part of the proof it follows from the boundary point lemma that $w_{\tau}$ cannot achieve the value 0 on the free boundary.

Lemma 3.5. Under the same assumptions as in Lemma 3.3 suppose that $\psi^{\prime}\left(\tau_{1}\right)=$ 0 and that the pair $\left(\psi\left(\tau_{1}\right), w\left(\cdot, \tau_{1}\right)\right)$ is not identically equal to the solution pair $(a, f)$ of $(\mathbf{S})$ in $\S 1$. Then there exists $\tau_{3} \in\left(\tau_{1}, \infty\right)$ such that $\psi^{\prime} \neq 0$ on $\left(\tau_{1}, \tau_{3}\right)$. Moreover, if $w_{\tau}\left(\cdot, \tau_{1}\right) \leq 0$ near $\eta=\psi\left(\tau_{1}\right)$, then $\psi^{\prime}>0$ on $\left(\tau_{1}, \tau_{3}\right)$, 
and if $w_{\tau}\left(\cdot, \tau_{1}\right) \geq 0$ near $\eta=\psi\left(\tau_{1}\right)$, then $\psi^{\prime}<0$ on $\left(\tau_{1}, \tau_{3}\right)$. Finally, for every $\tau_{-} \in\left(\tau_{2}, \tau_{1}\right)$ and every $\tau_{+} \in\left(\tau_{1}, \tau_{3}\right)$ we have $l_{\tau_{-}}>l_{\tau_{+}}$.

Proof. The statements concerning $w_{\tau}$ follow immediately from Lemma 3.4. It remains to examine the behaviour of $l_{\tau}$ near $\tau_{1}$. By standard maximum principle arguments, $l_{\tau}$ is nonincreasing on every $\tau$-interval in which $w_{\tau}$ stays away from zero on the free boundary, and, by the free boundary conditions again, this is now the case both on $\left(\tau_{2}, \tau_{1}\right)$ and $\left(\tau_{1}, \tau_{3}\right)$.

We claim that as $\tau$ crosses $\tau_{1}$, a drop in $l_{\tau}$ really must occur. It is no restriction to assume that $w_{\tau}\left(\eta_{1}, \tau_{1}\right) \neq 0$. Hence, possibly for some $\tau_{5}>\tau_{1}$, we have, by Lemma 3.4 again, that $w_{\tau} \neq 0$ on $\left\{(\eta, \tau): \eta_{1} \leq \eta \leq \psi(\tau), \tau_{1}<\tau \leq \tau_{5}\right\}$, so $w_{\tau}(\cdot, \tau)$ cannot have any sign changes on $\left[\eta_{1}, \psi(\tau)\right)$ if $\tau \in\left(\tau_{1}, \tau_{5}\right)$. On $\left[0, \eta_{1}\right]$, at least in a small neighbourhood of $\tau_{1}$, the number of sign changes of $w_{\tau}(\cdot, \tau)$ is nonincreasing, because $w_{\tau}\left(\eta_{1}, \tau_{1}\right) \neq 0$ (see [M]). We shall complete the proof by showing that as $\tau \uparrow \tau_{1}$, the number of sign changes of $w_{\tau}(\cdot, \tau)$ in $\left[\eta_{1}, \psi(\tau)\right)$ has to remain strictly positive.

Suppose not; then there exists $\tau_{6} \in\left(\tau_{2}, \tau_{1}\right)$, such that $w_{\tau}$ does not change sign on $\left\{(\eta, \tau): \eta_{1} \leq \eta \leq \psi(\tau), \tau_{6}<\tau<\tau_{1}\right\}$. Since clearly $w_{\tau} \not \equiv 0$, it follows that $w_{\tau} \neq 0$ on $\left\{(\eta, \tau): \eta_{1} \leq \eta \leq \psi(\tau), \tau_{6}<\tau<\tau_{1}\right\}$. Just as in the proof of Lemma 3.4 this gives a contradiction in $\left(\psi\left(\tau_{1}\right), \tau_{1}\right)$ in view of the boundary point lemma and (3.4)-(3.5).

Proof of Theorem 3.1. This is now obvious. By Lemma 3.4 the number of times that $\psi^{\prime}$ may become zero is limited. Hence, eventually $\psi^{\prime}$ has a fixed sign, so eventually $\psi(\tau)$ is monotone. Because of Lemma 2.5 this suffices.

\section{CONVERgenCe to EQUilibrium}

Theorem 4.1. $\lim _{\tau \rightarrow \infty} \psi(\tau)=a$.

Proof. By Theorem 3.1 the limit exists. Suppose

$$
L=\lim _{\tau \rightarrow \infty} \psi(\tau)>a .
$$

Choose $b \in(a, L)$. Then for $\tau$ sufficiently large we have $\psi(\tau)>b$, so $w(b, \tau)<0$. Hence we can use solutions of the $w$-equation with zero boundary data at $\eta=b$ as supersolutions for $w$. Now it is straightforward to check [CD] that the eigenvalue problem

$$
\left\{\begin{array}{l}
\varphi^{\prime \prime}+\frac{1}{2} \varphi=\frac{1}{2} \eta \varphi^{\prime}+\lambda \varphi \quad \text { for } 0 \leq \eta \leq b \\
\varphi^{\prime}(0)=\varphi(b)=0
\end{array}\right.
$$

has, up to a constant, exactly one positive solution $\varphi$ and that $\lambda>0$ because $b>a$. Hence for sufficiently small $\varepsilon>0$ the function

$$
\bar{w}(\eta, \tau)=-\varepsilon e^{\lambda \tau} \varphi(\eta)
$$

is a supersolution for $w$ if $\tau$ is large, implying that $w$ is unbounded, which is a contradiction.

Now suppose that

$$
L=\lim _{\tau \rightarrow \infty} \psi(\tau)<a
$$


Choose $b \in(L, a)$. Then for $\tau$ sufficiently large we have $\psi(\tau)<b$. Now we can use solutions of the $w$-equation with zero boundary data at $\eta=b$ as a subsolution. Again it is straightforward to check that $\lambda<0$ because $b<a$. Hence for sufficiently large $M>0$ the function

$$
\underline{w}(\eta, \tau)=-M e^{\lambda \tau} \varphi(\eta)
$$

is a subsolution for $w$ if $\tau$ is large, implying that $w(\cdot, \tau) \rightarrow 0$, which is a contradiction.

Proof of Theorem 1.1. By Theorems 3.1 and 4.1 there are two cases to consider. Either $\psi(\tau) \downarrow a$ or $\psi(\tau) \uparrow a$ as $\tau \rightarrow \infty$.

In the first case we have that $\psi(\tau)>a$. Hence if we define the function $V$ by

$$
-V(w)=\sup \{\mu>0: \mu f(\eta) \geq w(\eta) \forall \eta \in[0, a]\},
$$

for all $w \in C([0, \psi])$ with integral -1 , then clearly $v(\tau)=V(w(\cdot, \tau))$ is well defined and $-v(\tau) \leq 1$. In fact $-v(\tau)<1$, because $-v(\tau)=1$ would imply that $w(\cdot, \tau)$ coincides with $f$.

It follows from the Strong Maximum Principle that $v(\tau)$ is strictly decreasing. Thus $V$ is a strict Liapounov functional, continuous with respect to the supremum norm, and bounded from below. Also every solution orbit is compact with respect to this norm. Hence by standard Liapounov theory (see, e.g., [D]), Theorem 1.1 follows.

The case $\psi(\tau) \uparrow a$ is similar. Define

$$
v(\tau)=\inf \{\mu>0: \mu f(\eta) \leq w(\eta) \forall \eta \in[0, \psi(\tau)]\},
$$

and reason as before.

\section{REFERENCES}

[BCN] H. Berestycki, L. A. Caffarelli, and L. Nirenberg, Uniform estimates for regularization of free boundary problems, A collection of papers dedicated to Mischa Cotlar (C. Sadovsky, ed.), Marcel Dekker, New York, 1990.

[BH] M. Bertsch and J. Hulshof, Regularity results for an elliptic-parabolic free boundary problem, Trans. Amer. Math. Soc. 297 (1986), 337-350.

[BL] J. D. Buckmaster and G. S. S. Ludford, Theory of laminar flames, Cambrige Univ. Press, New York, 1982.

[CV] L. A. Caffarelli and J. L. Vazquez, A free boundary problem for the heat equation arising in flame propagation, preprint.

[CD] E. A. Coddington and N. Levinson, Theory of ordinary equations, Tata McGraw-Hill, New York, 1972.

[D] C. M. Dafermos, Asymptotic behaviour of solutions of evolution equations, Nonlinear Evolution Equations (M. Crandall, ed.), Academic Press, New York, 1978.

[GH] R. Gianni and J. Hulshof, The semilinear heat equation with a Heaviside source term, European J. Appl. Math. 3 (1992), 367-379.

[GP] V. Guillemin and A. Pollack, Differential topology, Prentice Hall, Englewood Cliffs, NJ, 1974.

[HH] D. Hilhorst and J. Hulshof, An elliptic-parabolic problem in combustion theory: convergence to travelling waves, J. Nonlinear Anal. 17 (1991), 519-546. 
[H] J. Hulshof, An elliptic-parabolic free boundary problem: continuity of the interface, Proc. Roy. Soc. Edinburgh Sect. A 106 (1987), 327-339.

[M] H. Matano, Nonincrease of the lapnumber of a solution for a one dimensional semilinear parabolic equation, J. Fac. Sci. Univ. Tokyo Sect. IA Math. 29 (1982), 401-441.

Laboratoire d’Analyse Numerique, CNRS et Université de Paris-Sud, 91401 Orsay CÉdex, France

E-mail address: hilhorst@lanor matups.fr

Mathematical Institute, Leiden University, P.O. Box 9512, 2300 RA Leiden, The NETHERLANDS

E-mail address: hulshof@ri.leidenuniv.nl 